\title{
Influencia del sexo en la práctica deportiva. Biología de la mujer deportista
}

\section{Carmen León París}

Arbor CLXV, 650 (Febrero 2000), 249-263 pp.

Muchas son las diferencias que en cuanto a las características fisiológicas muestran los hombres frente a las mujeres. Estas diferencias fisiológicas afectan a la respuesta al ejercicio, y en consecuencia al diferente rendimiento deportivo. Diferencias en la composición corporal, el metabolismo del calcio y del hierro, el tamaño de órganos y aparatos, asi como las diferencias en la edad de maduración afectan al rendimiento y a los patrones de ejercicio físico en las mujeres jóvenes. Otros cambios, como el embarazo y la menstruación modifican también esta respuesta tanto, desde el punto de vista fisiológico, como psicológico. A estas diferencias debemos añadir los factores sociológicos, debido a que la incorporación de la mujer al mundo del deporte de elite es relativamente reciente, y no siempre cuenta con el apoyo de medios técnicos, económicos y de comunicación necesarios. Tanto las diferencias fisiológicas como las psicológicas que caracterizan a las mujeres persistirán, por lo tanto parece necesario un mejor conocimiento de las mismas para comprender y obtener mejores resultados desde el punto de vista del rendimiento deportivo.

\section{Diferencias fisiológicas: sexo cromosómico}

El sexo viene determinado por los cromosomas sexuales, y la información contenida en sus genes es la responsable de las diferencias fisiológicas y psicológicas entre hombres y mujeres. En estos cromosomas 
se localiza la información que determina las características morfológicas de los genitales, la masa muscular, el tamaño cardíaco, etc. El hecho de que estas características beneficien el rendimiento deportivo de aquellas mujeres que posean un cromosoma masculino, condujo en los años 60 a la realización de test de verificación del sexo cromosómico. En los inicios fueron sometidas a reconocimientos físicos, posteriormente se reemplazaron por análisis de los cuerpos de Barr, de mucosa bucal, y los más novedosos test de reacción de cadenas de la polimerasa, sin embargo, en todos podríamos obtener falsos positivos, como resultados de anomalías cromosómicas, lo que conllevaría a una retirada injusta de dichas mujeres. Además no está demostrado que aquellas mujeres que presenten un sexo cromosómico $X Y$ posean una ventaja en su rendimiento deportivo, debido a que a pesar de mostrar niveles más elevados de testosterona presentan un síndrome de insensibilidad a los andrógenos. Actualmente están pues eliminados estos «test» de determinación del sexo en las competiciones de pista y campo. El análisis de la orina durante la realización de los controles Anti-dopaje permitiría desenmascarar cualquier situación.

\section{Crecimiento y maduración}

Las mujeres presentan un proceso de maduración más rápido que los hombres, su pubertad comienza antes, entre los 10 y 13 años para las chicas y entre los 12 y 15 años para los chicos. Las diferencias entre los sexos en altura, peso corporal, perímetros y diámetros óseos no se presentan hasta el inicio de la pubertad. En la pubertad comienzan a desarrollarse mayores diferencias entre los sexos, básicamente asociadas a los cambios endocrinos. La secreción de testosterona por los testículos disminuye en el nacimiento y es restituida durante la pubertad, produciendo un incremento en anabolismo proteico muscular, óseo, y en otras partes del cuerpo. En la mujer cuando una cantidad suficiente de hormonas gonadotrópicas es secretada por parte de la hipófisis anterior, el ovario se desarrolla y comienza la secreción de estrógenos. Los estrógenos tiene una influencia significativa sobre el crecimiento corporal, la anchura de la pelvis, el tamaño de la mamas, y el depósito de grasa, especialmente en caderas y muslos. Además los estrógenos aumentan el nivel de crecimiento óseo, permitiendo que el último alargamiento óseo sea alcanzado entre los dos y cuatro años que siguen al inicio de la pubertad. En consecuencia, las mujeres crecerán muy rápidamente los primeros años después del inicio de la 


\section{Influencia del sexo en la práctica deportiva}

pubertad, y entonces cesará su crecimiento. Por el contrario, los varones presentan una fase de crecimiento más larga, lo que les permite alcanzar una mayor altura. Así pues, las adolescentes jóvenes son más altas que los chicos de su misma edad, sin embargo, los chicos les alcanzarán y sobrepasarán posteriormente. Además, los varones crecen durante más tiempo, lo que resultará finalmente en un mayor peso $(17 \%) \mathrm{y}$ tamaño corporal (10\%) de los varones frente a las mujeres.

La composición corporal cambia durante la pubertad, fundamentalmente debido al incremento en la secreción de las hormonas gonadotropas por parte de la hipófisis. Estos cambios hormonales conducen a un estimulo e incremento de las hormonas sexuales, estrógenos en la mujer y andrógenos en el varón. Los estrógenos aumentan el tejido adiposo, mientras que el efecto de los andrógenos es el incrementar el tejido libre de grasa. Los cambios hormonales que ocurren durante la pubertad son, en parte, los responsables del aumento de las diferencias en el rendimiento físico presentes entre chicos y chicas. Durante la pubertad las mujeres experimentan un incremento en su masa muscular, pero este incremento es considerablemente inferior al que muestran los varones. Los andrógenos poseen una importante función en la síntesis proteica. La testosterona en niños y niñas prepuberes presenta valores similares (20-60 ng. $\left.\mathrm{ml}^{-1}\right)$, durante la pubertad los valores de testosterona en el varón se incrementan hasta valores similares a los del adulto (600 ng. $\mathrm{ml}^{-1}$ ), mientras que en las mujeres las cifras permanecen en valores similares.

Las características antropométricas presentan bastantes similitudes entre chicos y chicas durante el periodo prepuber, hasta los 10 años ambos sexos poseen valores similares en longitud de pierna, circunferencia de brazo, peso corporal, talla sentado, e índice ponderal. A lo largo del crecimiento los varones presentan mayor diámetro epicondíleo de fémur y húmero, longitud de brazo, y perímetro de tórax y hombro. Sin embargo, las mujeres presentan un mayor diámetro de cadera con relación a la altura. Las medidas antropométricas experimentan un marcado cambio durante la pubertad, mientras que los chicos presentan un mayor desarrollo de los hombros, las mujeres experimentan dicho incremento en el diámetro de su cadera. El menor tamaño del hombro da como resultado un menor desarrollo de la fuerza en el miembro superior, mientras que el mayor perímetro de cadera hace que el ángulo femoral sea más prominente que en los varones, y produce un descenso del centro de gravedad. Esta situación unida al menor tamaño del miembro inferior femenino conceden a la mujer una mayor ventaja en aquellas actividades que requieran equilibrio, 
pero limitan otras confiriendo una desventaja biomecánica, como el salto de longitud, la carrera, o los lanzamientos. La mujer muestra una mayor flexibilidad debido a los niveles basales de la hormona relaxina.

\section{Composición corporal}

Los estudios muestran que durante la edad escolar las mujeres presentan más grasa y menos masa muscular que los chicos, de su edad. El porcentaje de grasa disminuye considerablemente con la actividad física. Sin embargo, no conviene olvidar que en el ideal de porcentaje de grasa interviene también los dictámenes de la moda, no siempre paralelos a los objetivos de la salud. Esta situación puede desembocar en un importante y serio problema, la anorexia nerviosa. Esta patología se encuadra dentro de la triada clásica de la mujer deportista ACSM: amenorrea, anorexia nerviosa y osteoporosis. Sin embargo, la anorexia nerviosa no es solo un problema en el ámbito deportivo, sino que su importancia fuera del mismo y para la sociedad en general ha llevado a nuestros gobernantes a tomar medidas. La grasa presente en el organismo se divide en esencial y no esencial. La denominada grasa esencial se localiza encuentra almacenada en el sistema nervios central, corazón, pulmones, y médula ósea, y posee una importante función en la conducción del impulso nervioso, estructura de las membranas celulares e interviene en el metabolismo y en la protección de diversos órganos frente a las agresiones traumáticas. En las mujeres la grasa sexo-específica está considerada esencial y se encuentra almacenada en el pecho y en el tejido subcutáneo de miembros inferiores, sobre todo en el entorno de la pelvis. Los varones presentan una distribución de la grasa con predominio de las zonas abdominales y las regiones superiores del cuerpo. Así frente a un $20 \%$ de esencial en los varones, en las mujeres la grasa esencial representa hasta un $40 \%$ de la grasa presente en el organismo. No se conoce con certeza el mínimo porcentaje de grasa esencial saludable, pero si se sabe que un porcentaje de grasa extremadamente bajo puede ocasionar consecuencias adversas sobre la salud. Es deseable un bajo nivel de grasa, ya que se ha visto que las grandes diferencias en el consumo máximo de oxígeno entre hombres y mujeres, se ven reducidas cuando dichos valores se expresan en valores relativos al peso libre de grasa. Sin embargo, es difícil valorar con exactitud ese mínimo porcentaje de grasa, además ese porcentaje es diferente para cada 


\section{Influencia del sexo en la práctica deportiva}

deporte, especialidad y momento de la temporada. Así pues, en los deportes de resistencia, como las corredoras de fondo son deseables bajos niveles de grasa, las nadadoras podrían encontrar ventaja en un mayor porcentaje de grasa, lo que les permite arrastrar menos peso y ser más eficientes para este deporte. Queda la duda de sí podrían nadar más rápido si fueran capaces de desarrollar la fuerza de los hombres. Las diferencias en la composición corporal afectan a la capacidad de realización del ejercicio físico y del rendimiento de las mujeres, suponiendo una desventaja en aquellos deportes que deben movilizar su masa corporal contra la gravedad, como la carrera, el salto, la trepa; por otra parte esta mayor masa grasa también supone una desventaja en el proceso de liberación del calor corporal durante el ejercicio. Mientras que el aumento de tejido adiposo en las corredoras de fondo no parece suponer una mejora en el metabolismo graso y por lo tanto en su rendimiento, si parece suponer una ventaja en el rendimiento en las mujeres nadadoras de larga distancia ${ }^{12}$.

Con la edad tanto varones como mujeres experimentan un incremento en el acumulo de grasas, debido fundamentalmente al menor nivel de actividad física, menores niveles de testosterona, mientras que la cantidad de calorías ingeridas apenas experimenta modificaciones.

También la cantidad y distribución de los fluidos corporales experimentan diferencias debidas al sexo. Las diferencias debidas al sexo se hacen evidentes a partir de los 18 años, cuando el agua corporal total de las mujeres puede descender considerablemente, diferencia debida casi totalmente a la caída del liquido intracelular hasta un $23 \%$, debido a que el mayor peso graso contiene solo una pequeña cantidad de agua en el tejido adiposo. Por otra parte es importante tener en cuenta el incremento de peso que las mujeres presentan durante la fase premenstrual debido a un incremento en la retención de líquidos.

\section{Transporte de oxígeno y resistencia}

El consumo de oxígeno máximo $\left(\mathrm{VO}_{2}\right.$ máx $)$ está considerado como el mejor índice de capacidad de resistencia cardiorrespiratoria. La capacidad para el transporte de oxígeno viene determinada genéticamente, y las diferencias se deben más al nivel de entrenamiento y a los potenciales biológicos que a las diferencias debidas al sexo. Antes de la pubertad no hay diferencias entre el $\mathrm{VO}_{2 \text { máx }}$ en chicos y chicas, cuando se expresan en valores relativos al peso. Las mujeres tienden a alcanzar su $\mathrm{VO}_{2 \text { máx }}$ entre los 13 y 15 años, mientras que los varones lo hacen 
entre los 18 y 22 años. Cuando hablamos de valores' de $\mathrm{V}_{\mathrm{O} 2 \text { pico }}$ es muy importante clarificar el nivel de entrenamiento de sus componentes, ya que las mujeres entrenadas presentan valores de este parámetro superiores a los varones con un menor nivel de condición física.

En general se establecen mayores diferencias en .cuanto a la capacidad de resistencia y transporte de oxígeno en aquellos deportes que implican resistencia del miembro superior. El tamaño de los órganos y la masa corporal son importantes en la determinación de diferencias debidas al sexo en el rendimiento de resistencia. El mayor tamaño proporciona una mayor capacidad de potencia. Los varones presentan mayor porcentaje de masa muscular tanto en términos absolutos como relativos, mientras que las mujeres son superiores en el porcentaje de masa ósea. La mayor masa libre de grasa representa una ventaja en los deportes de resistencia. La composición de las fibras musculares, tanto fibras de contracción rápida como las de contracción lenta, es similar en varones y mujeres. Sin embargo, tanto las fibras de contracción rápida como las lentas son de mayor tamaño en los hombres. Sin embargo, algunos estudios han hallado un mayor porcentaje de fibras rápidas en los varones. Las diferencias debidas al sexo en el rendimiento de resistencia parecen aumentar a medida que disminuye el nivel deportivo, Así pues, las diferencias entre hombres y mujeres en la elite son menores que las encontradas en los deportistas de niveles más bajos, probablemente en este hecho influyan los similares beneficios obtenidos por el entrenamiento, y los similares sistemas de entrenamiento aplicados hoy en día a hombres y mujeres. Las diferencias observadas en el rendimiento de resistencia se deben fundamentalmente a las diferencias presentadas en la fuerza y la potencia. Hombres y mujeres presentan las mismas mejoras relativas cuando son sometidas a los mismos estímulos de entrenamiento. Los programas de entrenamiento no muestran grandes diferencias entre hombres y mujeres de elite, y están cada vez más próximos en intensidad.

El consumo de oxígeno en valores absolutos es un $40 \%$ superior en los hombres frente a las mujeres, cuando hablamos de valores relativos al peso este porcentaje desciende hasta el $20 \%$, y finalmente las diferencias son de solo un $10 \%$ cuando los valores de consumo de oxígeno se expresan en valores relativos al peso libre de grasa. Aunque el porcentaje de grasa es un handicap para la mujer en los deportes de resistencia, no es el responsable de todas las diferencias debidas al sexo que se observan en el rendimiento. Algunos investigadores han concedido demasiada responsabilidad, hasta un $70 \%$ al peso graso, mientras que el mayor consumo máximo de oxígeno lo hace solo en 


\section{Influencia del sexo en la práctica deportiva}

un $20 \%$. El consumo máximo de oxígeno está directamente en relación con el tejido activo implicado en dicho ejercicio. Podemos concluir que este depósito graso esencial dependiente del sexo es el mayor determinante de las diferencias en la respuesta metabólica a la carrera, y por lo tanto de las diferencias en el rendimiento deportivo. En general, los hombres poseen un mayor tamaño y volumen cardíaco que las mujeres, tanto en términos absolutos como relativos. Esto resulta en un mayor gasto cardíaco durante el ejercicio, lo que contribuye notablemente a las diferencias halladas en el consumo máximo de oxígeno. Incluso aunque las mujeres presenten una mayor frecuencia cardíaca durante un ejercicio no es suficiente para compensar su menor volumen sistólico. El menor gasto cardíaco resultante contribuye a su menor capacidad aeróbica. La concentración de hemoglobina $(\mathrm{Hb})$ es también más elevada en los varones, lo que contribuye notablemente a su mayor capacidad de transporte de oxígeno (13.7 frente a $15.8 \mathrm{gr} / 100 \mathrm{ml})$. Estas diferencias son atribuibles a varios factores entre los que debemos destacar el efecto de los andrógenos sobre la producción de $\mathrm{Hb}$, las pérdidas que acompañan al ciclo menstrual y las deficiencias en la ingesta calórica. Los estudios que han analizado el gasto calórico de la carrera muestran diferentes conclusiones así mientras unos indican que el varón es más económico por su mayor masa libre de grasa, resulta extremadamente difícil comparar los datos de hombres y mujeres de diferente nivel de condición física, antecedentes de entrenamiento y rendimiento en carrera. Bosco y $\operatorname{col}^{4}$ han mostrado que el coste energético de la carrera está en relación con el porcentaje de fibras rápidas. Dado que la mayoría de las corredoras presentan una mayor proporción de fibras lentas, contarían con una predisposición a una mayor economía de carrera, sin embargo el hecho se contrarresta por su mayor porcentaje de masa grasa. Por el contrario otros investigadores no han hallado diferencias debidas al sexo en la distribución de las fibras musculares. Saltin y col. ${ }^{11}$ encontraron que los hombres poseían unos valores más extremos en las distribución de las fibras, lo que podría ser significativo a la hora de tener en cuenta las diferencias debidas al sexo. No se han encontrado diferencias debidas al sexo en los efectos del entrenamiento sobre la mejora de la capacidad $\mathrm{VO}_{2 \operatorname{má}}$. El VO ${ }^{2 m a ́ x}$ puede aumentar hasta un $20 \%$ en ambos sexos. La frecuencia cardíaca de reposo y submáxima es más elevada en las mujeres, dado que los requerimientos de oxígeno por $\mathrm{Kg}$ de peso durante el ejercicio son los mismos para hombres y mujeres, el corazón de la mujeres debe latir más rápido para compensar su menor volumen sistólico, es decir, su menor capacidad de bombeo, además hay que añadir el mayor 
volumen sanguíneo que presentan los varones. La menor diferencia arteriovenosa de oxigeno encontrada en la mujeres, es el resultado de los menores niveles de hemoglobina presentes en las mujeres. En los sujetos con nivel de entrenamiento similar existen pocas diferencias en la frecuencia cardíaca máxima entre ambos sexos. Debido al mayor gasto cardíaco máximo los hombres presentan una mayor presión sistólica durante el ejercicio. La recuperación de la frecuencia cardíaca es más lenta en las mujeres, lo cual debe ser tenido en cuenta por los entrenadores para programar los periodos de recuperación entre las distintas cargas de trabajo.

Paralelamente las diferencias encontradas en la respuesta respiratoria son también debidas al tamaño de los órganos. Las mujeres suelen presentar una frecuencia ventilatoria ligeramente superior para la misma intensidad de trabajo, probablemente se deba a que para la mujer esa carga le supone trabajar a un porcentaje superior de su máximo consumo de oxígeno. Los volúmenes ventilatorios, incluyendo el volumen tidal son ligeramente inferiores en las mujeres, en parámetros máximos y submáximos. Así pues, mientras que los varones pueden alcanzar volúmenes de ventilación máxima de hasta 250 litros por minuto, las mujeres no suelen alcanzar niveles tan elevados. Esta podría considerarse como otra de las causas de limitación del rendimiento en las mujeres deportistas de elite.

\section{Rendimiento físico}

Los hombres poseen aproximadamente un $20 \%$ más que las mujeres en su capacidad de resistencia y también para el ejercicio de corta duración y elevada intensidad. Esto es debido a los factores ya comentados, como las diferencias en el peso corporal, así como en el tamaño corporal y de las vísceras, como el corazón. Las diferencias en el rendimiento deportivo debidas al sexo se muestran más acusadas en aquellas actividades que se benefician de la fuerza y la talla, como el levantamiento de peso y la velocidad. Las diferencias debidas al sexo en la natación son menores, entre un $7-13 \%$ debido a la capacidad para mantenerse a flote. Sin embargo, estudios recientes utilizando el centro de masa y el centro de flote no parecen mostrar esa desventaja en lós varones ${ }^{10}$.

Los chicos son mejores en deportes de «sprint», salto altura, longitud, y bastante mejores en lanzamientos. En la pubertad las diferencias debidas al sexo se hacen mayores, los chicos muestran un mayor grado 
de mejora que las chicas. Hombres y mujeres responden de forma similar a todos los tipos de entrenamiento físico. Sin embargo, en la literatura se muestran resultados contradictorios. Cuando se evalúan estos resultados es necesario tener en cuenta el nivel de partida de los sujetos que forman parte del estudio. Algunos de los trabajos revisados muestran una mejora en el rendimiento superior en mujeres que en hombres, pero esto generalmente es debido al relativo mayor nivel inicial de los hombres en cuanto a sus parámetros de forma física. Los hombres están generalmente más cerca del potencialmente máximo nivel. La mayoría de los estudios han mostrado una respuesta al entrenamiento igual en hombres y mujeres.

Respecto a la regulación de la temperatura durante el ejercicio también hay muchos tópicos. Los primeros estudios hallaron que las mujeres toleraban mejor el ejercicio físico realizado en condiciones de calor, pero generalmente estos estudios utilizaban poblaciones de hombres y mujeres sedentarios. En los estudios realizados con mujeres entrenadas, los niveles de sudoración durante el ejercicio eran generalmente menores. La aclimatación y el control de la temperatura corporal era similar en mujeres y hombres. Los hombres al igual que las mujeres dependen de mecanismos circulatorios, como el control del tono vascular, para controlar la temperatura. El nivel de forma física, más que las diferencias debidas al sexo, son importantes en la determinación de la tolerancia al calor y de la capacidad de aclimatarse. Las mujeres bien entrenadas toleran la hipóxia, la altitud y el calor al mismo nivel que los sujetos varones entrenados.

\section{Metabolismo muscular}

En hombres y mujeres con nivel de entrenamiento similar el glucógeno muscular, el lactato sanguíneo en ejercicio máximo, la capacidad metabólica de las grasas y composición de las fibras musculares son similares. Algunos aspectos de la bioquímica no responden al entrenamiento también como en los hombres, por ejemplo la "síntesis de glucógeno y la capacidad de oxidación de las grasas. La actividad de las enzimas succinato deshidrogenasa y carnitin palmitoil transferasa son mayores en hombres. La producción de glucógeno muscular es estimulada por la testosterona, cuya concentración es superior en varones. Algunos autores han propuesto la hipótesis de que las mujeres son superiores en los deportes de resistencia, dado su mayor porcentaje de grasa y su capacidad de metabolizarla más efectivamente que los 
hombres. Esta hipótesis partió de observaciones empíricas extraídas de los corredoras de maratón femeninas. Algunas de estas atletas dicen no experimentar la fatiga extrema que notan los hombres al final de la carrera. Esta extrema fatiga conocida como el «muro» se caracteriza por un vaciamiento completo de los depósitos de glucógeno hepático y muscular. Las investigaciones, sin embargo, no apoyaban esta hipótesis. Incluso el más magro de los atletas tiene amplios depósitos de grasa. Muchos estudios han mostrado en los varones una capacidad ligeramente superior para metabolizar las grasas.

Los chicos son más fuertes durante la infancia y adolescencia que las chicas. En la infancia el peso corporal y la masa muscular son similares en chicos y chicas, sin embargo, incluso entonces el músculo de los varones es capaz de desarrollar algo más de tensión por área de sección transversal. En el adulto los hombres son hasta un $40 \%$ superiores a las mujeres en la mayoría de los grupos musculares. Estas diferencias en la fuerza son algo mayores en miembros superiores. Los varones tienen una mayor proporción de su masa en la mitad superior del cuerpo, lo cual les confiere una ventaja. La mayoría de los estudios muestran que hombres y mujeres desarrollan una fuerza similar por área de sección transversal del músculo. El promedio de la fuerza desarrollada por hombres y mujeres es de $6 \mathrm{Kg} . \mathrm{cm}^{-2}$. Algunos estudios, sin embargo, han mostrado un fuerza ligeramente superior en los hombres. Las fibras musculares son similares en hombres y mujeres en cuanto a su distribución y características histoquímicas, tanto en deportistas como en sedentarios. Todas las fibras musculares presentan menor área sección transversal en las mujeres. La mayoría de los estudios muestran igual numero de fibras en los músculos de varones y hembras, aunque algunos estudios han hallado mayor número de fibras musculares en el bíceps de varones sedentarios, en comparación con las mujeres sedentarias. Los niveles de andrógenos superiores en los varones son en parte los responsables de las diferencias encontradas en los niveles de fuerza entre hombres y mujeres. Los andrógenos son unás potentes hormonas anabólicas responsables de la mayor parte de la hipertrofia muscular encontrada en los varones durante el crecimiento puberal. Algunos autores apuntan la hipótesis de que debido a los bajos niveles de testosterona presentes en la mujer, estas no presentan un desarrollo de musculos mayores con el entrenamiento, esta hipótesis viene apoyada por algunos estudios que no han hallado que el entrenamiento de fuerza proporcione cambios en el área de sección transversal de los músculos de las mujeres con el entrenamiento ${ }^{2}$. Sin embargo, otros estudios no apoyan esta teoría 


\section{Influencia del sexo en la práctica deportiva}

debido a que en ellos se han presentado cambios relativos en la fuerza similares entre hombres y mujeres sometidos a los mismos programas de entrenamiento. Estudios más recientes en los que se utiliza tecnología más sofisticada como la Tomografía computerizada y los Ultrasonidos nos permiten realizar mediciones de forma exacta. En estos estudios se ha visto que los cambios en la sección transversal son similares al aplicar programas de entrenamiento similares. Los cambios tempranos que acontecen con los programas de entrenamiento de peso son debidos a factores neurales, incluyendo una mejora en el patrón de reclutamiento de unidades motoras. Los cambios en el tamaño de la fibra muscular necesitan mucho más tiempo para desarollarse. La falta de estudios a más largo plazo hace prematuro establecer que el potencial para la hipertrofia muscular sea igual en hombres y mujeres. Durante los primeros meses de entrenamiento los incrementos observados en el tamaño muscular son relativamente los mismos en hombres y mujeres. Sin embargo, las diferencias debidas al sexo en la capacidad máxima para alcanzar la hipertrofia muscular permanecen aún por conocer. Aunque la diferencias en los parámetros de fuerza entre hombres y mujeres se reducen cuando expresamos dichos vảlores respecto al peso magro, lo cual indica que las cualidades histológicas y bioquímicas del músculo y sus propiedades de control motor no muestran diferencias entre sexos, persisten ciertas diferencias entre miembro superior e inferior. Esto podría deberse a que las mujeres poseen un mayor porcentaje de masa magra por debajo de la cintura, así como al mayor uso de esta masa muscular, comparado con los patrones masculinos. Gracias a la utilización de las biopsia musculares a partir de los años sesenta ha sido posible identificar los diferentes tipos de fibras musculares y su repercusión sobre el rendimiento en diferentes deportes. La clasificación usada más frecuentemente las divide en tres tipo, las fibras de contracción lenta u oxidativas (Tipo I), las fibras de contracción rápida oxidativa (Tipo IIa), y la fibras de contracción rápida glucolítica (Tipo IIb). Como indicábamos anteriormente estos estudios mostraron que a pesar de que hombres y mujeres que practicaban un mismo deporte, poseían una distribución similar del tipo de fibras, los varones mostraron valores más extremos en sus porcentajes de distribución. Estudios recientes ${ }^{7}$ sobre medición de la fuerza en hombres y mujeres no hallado diferencias en condiciones estáticas de ejercicios de fuerza, sin embargo, el ejercicio dinámico si ha mostrado diferencias debidas al sexo. Estas diferencias tienen importantes implicaciones para los modelos biomecánicos de fuerza. Los estudios más recientes de Schultz y Perri ${ }^{13}$ utilizando regiștros electromiográficos 
(EMG) han analizado la fase de reclutamiento de la activación neuromuscular refleja después de someter a la rodilla a una carga de estrés, y han hallado pequeñas diferencia en algunos aspectos de la respuesta neuromuscular. Son necesarias más investigaciones para explorar las diferencias en esta respuesta y su papel potencial como factores predisponentes en la mayor incidencia de patologías de rodilla, como la rotura del ligamento cruzado anterior debido a su efecto sobre la estabilidad dinámica articular ${ }^{13}$.

\section{Hormonas femeninas y densidad ósea}

La liberación de las hormonas gonadotropas desde el hipotálamo y la pituitaria desencadena la menarquia o primera menstruación. El retraso en la aparición de la primera regla está influenciado por factores genéticos, así como por el entrenamiento. La amenorrea, ausencia o irregularidad de la menstruación, es un hecho que ocurre con mayor frecuencia en las deportistas que en la población sedentaria. El ciclo menstrual está regulada por una serie de hormonas, los estrógenos se producen continuamente durante el ciclo, en baja cantidad durante el comienzo de la fase folicular, aumentando hasta un pico a lo largo de la fase folicular y desencadenando la ovulación. Durante la fase lútea el incremento en los estrógenos causa el engrosamiento y maduración del endometrio. Por su parte la progesterona ayuda en el proceso de maduración y favorece los cambios que debe sufrir el endométrio para convertirse en una estructura secretora preparada para la implantación del óvulo fecundado. Al final de la fase lútea las concentraciones de estrógenos y progesterona disminuyen, desencadenando la menstruación por una rotura del endométrio. La hormona liberadora de gonadotrofinas ( $\mathrm{GnRH})$ es secretada por el hipotálamo y controla la secreción pituitaria de las hormonas foliculoestimulante (FSH) y luteinizante (LH). La FSH estimula el crecimiento del folículo ovárico y la producción de estrógenos. Cuando los estrógenos alcanzan un nivel crítico se libera la $\mathrm{LH}$, la liberación de $\mathrm{LH}$ desencadena la ovulación y está implicada en la transformación del folículo en cuerpo lúteo. El cuerpo lúteo produce estrógenos y progesterona.

Algunos factores, como el estrés y una dieta pobre, pueden alterar el ciclo menstrual, y además el entrenamiento podría empeorar la situación de estos problemas preexistentes. El entrenamiento de alto volumen e intensidad puede aumentar la incidencia de amenorrea. Las mujeres con historia previa de irregularidades menstruales son 


\section{Influencia del sexo en la práctica deportiva}

más propensas a presentar amenorrea en relación con el entrenamiento. La composición corporal puede influir en el ciclo menstrual, sin embargo, a pesar de que la grasa ocupa un lugar importante en la interconversión de estrógenos, algunos estudios no han podido encontrar una relación entre la composición corporal y la falta de ciclos menstruales.

Las causas psicológicas implicadas en la amenorrea secundaria no están completamente comprendidas. El desencadenante inicial podría ser una disminución en la secreción de GnRH y $\mathrm{LH}$ El entrenamiento de alta intensidad aumenta los niveles de hormonas como el cortisol, opioides endógenos y prolactina, las cuales producen una supresión de la secreción de GnRH y LH. El ejercicio físico se acompaña además de un incremento en la secreción de catecolaminas, que inhibe la producción de $\mathrm{GnRH}$ y conduce a una reducción en los niveles de estrógenos, resultando en una alteración del ciclo menstrual. Otros factores como la edad de la menarquia, el estrés físico, la ingesta calórica y de calcio, y los desordenes alimenticios han sido relacionados con las alteraciones del ciclo menstrual. El cese de la función menstrual, amenorrea, podría reflejar la incapacidad para adaptarse al estrés del estilo de vida y de los factores ambientales asociados al entrenamiento y la competición ${ }^{5}$. En 1994 Sundgot-Borgen ${ }^{14}$ publicó un articulo en el que citaba entre otros los siguientes factores desencadenantes: periodos prolongados de dieta, frecuentes fluctuaciones de peso, repentino incremento en el volumen de entrenamiento, suceso traumáticos, lesiones, y la pérdida o cambio de entrenador. Puede incluirse dentro de una peligrosa triada ${ }^{1}$, cuyos síntomas son: amenorrea, desordenes alimenticios y osteoporosis. Los estudios encaminados a analizar las practicas nutricionales de las atletas han hallado que en la mayoría de las mujeres la ingesta de energía es inadecuada para su carga de entrenamiento, calculada en base a la intensidad, duración, y frecuencia. Además las dosis de algunos minerales y oligoelementos, entre ellos el hierro, calcio, vitamina B12 y el zinc, están por debajo de las dosis diarias recomendadas. estos hallazgo, sin embargo, no han sido hallados en los varones, los cuales ingieren una cantidad adecuada de energía para su gasto energético diario. Según Hawley y col. ${ }^{8}$ un incremento en la cantidad de hidratos de carbono por parte de estas atletas, podría mejorar la capacidad de entrenamiento y los procesos de recuperación de las actividades de elevada intensidad en las mujeres. La causa primaria de utilización de píldoras anticonceptivas oral (AO) es la contracepción, y la segunda la manipulación del ciclo menstrual para controlar los síntomas premenstruales, así como la coincidencia en el tiempo de menstruación y una competición deportiva. El efecto de los 
AO sobre la densidad ósea en la mujeres menstruantes no está claro, algunos estudios han mostrado efectos positivos, otros negativos y otros no han hallado efectos con el uso de AO sobre el rendimiento deportivo. En las mujeres atletas parece que mejora la densidad ósea, no estando claros los efectos hallados sobre la fracturas de estrés y las lesiones de tejidos blandos. Los efectos de los AO sobre el rendimiento son particularmente relevantes en las deportistas de elite. Aunque algunos estudios han hallado una disminución en el consumo máximo de oxígeno, esto no siempre se refleja sobre el rendimiento en la pista. Incluso en algunas ocasiones su utilización podría incrementar el rendimiento, por sus efectos reduciendo la intensidad de los síntomas premenstruales, y disminuyendo las pérdidas sanguíneas. Conviene conocer los cambios en la fuerza y en la percepción del rendimiento que las mujeres experimentan durante las diferentes fases del ciclo menstrual. El trabajo realizado por Jacobson y Lentz ${ }^{9}$, en las cuatro fases diferentes del ciclo menstrual, menstruación, fase estrogénica, ovulación, y fase progestágena, encontraron un menor desarrollo de la fuerza durante la fase premenstrual, asi como una menor sensación de la fuerza y la potencia. Además las deportistas también mostraron una menor potencia durante la fase menstrual. Esto nos muestra los cambios en el rendimiento y en la percepción del rendimiento en la fase premenstrual. Los efectos perjudiciales como el incremento de peso no parecen tener lugar utilizando las $\mathrm{AO}$ actuales que incluyen pequeñas dosis de estrógenos y progestágenos. La ventajas, por tanto, podrían superar a las desventajas, y dado que existe una variación individual en la respuesta a los $\mathrm{AO}$, la decisión final debe ajustarse a criterios individuales ${ }^{3}$. Los efectos beneficios del estradiol, uno de las hormonas estrogénicas femeninas, son sin duda alguna su protección frente a las patologías cardiovasculares, pero desde el punto de vista deportivo merece la atención señalar su efecto limitador de la liberación de creatinquinasa (CK), sin embargo, a pesar de producir un freno en la liberación de CK no se ha podido demostrar aún que este directamente relacionado con un menor daño muscular ${ }^{6}$. Las consecuencias de la amenorrea incluyen entre otros: infertilidad, dificultad para alcanzar el pico de masa ósea, perdida prematura de hueso, fracturas de estrés y escoliosis. La fertilidad puede verse afectada en situaciones de sobreentrenamiento, sin embargo, habitualmente tanto los ciclos menstruales regulares como la fertilidad se restauran con el reposo y la mejora dietética, debido a que los efectos del ejercicio sobre la fertilidad son de carácter temporal. La amenorrea puede causar osteoporosis debido a la supresión de los niveles de estrógenos. La osteoporosis es 
la pérdida de minerales óseos, las atletas jóvenes experimentan una disminución de la densidad ósea y una dificultad para alcanzar el pico de densidad ósea prevista. La incidencia de fracturas de estrés es superior entre las mujeres atletas amenorreicas frente a las que presentan ciclos normales, siendo el bajo nivel de estrógenos la causa de la fragilidad ósea en este grupo de mujeres. La amenorrea puede suponer un problema en la mujer atleta si presenta sobreentrenamiento. Por otra parte el ejercicio físico y la actividad regular controlada contribuyen a la prevención de fracturas.

\section{Bibliografía}

1 ACSM American College of Sports Medicine (1997): The female athlete triade. Med. Sci. Sports Exerc. 29(5), i-ix.

2 BAILEY, L.L.; BYRNES, W.C.; Dickinson, A.L. y Foster, V.L. (1987): Muscle hypertrophy in women following a concentrated resistance training program. Med. Sci. Sports Exerc. 19(suppl), S16.

3 Bennell, K.; White, S. y CRossley, K. (1999): The oral contraceptive pill: a revolution for sport women? Brithish J. of Sports Med. 33(4), 231-238.

4 Bosco, C. et al. (1987): Relationship between the efficiency of muscular work during jumping and the energetics of running. Eur. J. Appl. Physiol. 56, 138-143.

5 DUECK, C.A.; MANORE, H.M. y MATT, K.J. (1996): Role of the energy balance in athlete menstrual dysfunction. Int. J. Sports Nutrition 6(2), 165-190.

6 Dumke, C.L. (1996): Protective mechanism of estradiol on eccentrically induced muscle damage. Microfirm Publications Int'l Inst. for Sport and Human Performance. University opf Oregon, Eugene, 1 microficha (82 fr), abstract.

7 FOTHERGILl, D.M.; GRIEVE, D.W. y PINDER, A.D.J. (1996): The influence of task resistance on the characteristics of maximal one and two-handel lifting exertions in men and women. Eur. J. Appl. Physiol. And Occup. Physiol. 72 (5/6), 430-439.

8 HaWley, S.; Dennis, S.C.; LindSAY, F.H. y NoAKES, T.D. (1995): Nutricional practics of athletes: are they sub-optimal? Journal of Sport Sci. 13, S75-S81.

9 JACOBSON, B.H. y LENTZ, W. (1999): Strenght and perceived performance differences during four phases of the menstrual cycle. Int. J. Sport Med. 3, 1-13.

10 MC LEAN, y HINRICHS, R.N. (1998): Sex differences in the centre of buoyancy location of competitive swimmers. Journal of Sport Sci. 16(4), 373-383.

11 SAltin, B.; Hakkinen, J.; KaUHANEN, H. y Komi, P.V. (1977): Fiber types and metabolic potentials of skeletal muscles in sedentary man and endurance runners. Ann. NY Aca. Sci. 301, 3-29.

12 Sharp, C. (1984): Physiology and the woman athlete. New Scientist August, 22-24.

13 ShULTZ, S.J. y PERRIN, D.H. (1999): Using surface EMG to asses sex differences in neuromuscular response characteristics. Journal of Athletic Training 34(2), 165-176.

14 SUNGODT-BORGEN, J. (1994): Risk and trigger factors for developmen featuring disorders in female athletes. Med. Sci. Sports Exerc. 26(4), 414-419. 